\title{
RESEARCH
}

Open Access

\section{Soluble epoxide hydrolase modulates immune responses in activated astrocytes involving regulation of STAT3 activity}

Chia-Chi Hung ${ }^{1,3+}$, Yi-Hsuan Lee ${ }^{1 \dagger}$, Yi-Min Kuo ${ }^{1}$, Pei-Chien Hsu', Huey-Jen Tsay², Ying-Ting Hsu², Chih-Chin Lee ${ }^{3}$, Jia-Jun Liang ${ }^{3}$ and Feng-Shiun Shie ${ }^{3 *}$

\begin{abstract}
Background: Astrocyte activation is a common pathological feature in many brain diseases with neuroinflammation, and revealing the underlying mechanisms might shed light on the regulatory processes of the diseases. Recently, soluble epoxide hydrolase (sEH) has been proposed to affect neuroinflammation in brain injuries. However, the roles of astrocytic SEH in brains with neurodegeneration remain unclear.

Methods: The expression of astrocytic sEH in the brains of APPswe/PSEN1dE9 (APP/PS1) mice developing Alzheimer's disease (AD)-like pathology was evaluated by confocal imaging. LPS-activated primary astrocytes with mRNA silencing or overexpression of $\mathrm{sEH}$ were used to investigate its regulatory roles in astrocyte activation and the induction of proinflammatory markers. Primary astrocytes isolated from a sEH knockout $\left(\mathrm{sEH}^{-/-}\right)$background were also applied.

Results: The immunoreactivity of SEH was increased in activated astrocytes in parallel with the progression of AD in APP/PS1 mice. Our data from primary astrocyte cultures further demonstrate that the overexpression of sEH ameliorated, while the silencing of sEH mRNA enhanced, the lipopolysaccharides (LPS)-induced expression of pro-inflammatory markers, such as inducible nitric oxide, cyclooxygenase 2 (COX-2), and pro-inflammatory cytokines. These findings suggest that $\mathrm{SEH}$ negatively regulates astrocyte immune responses. Enhanced immune responses found in $\mathrm{LPS}^{-a c t i v a t e d ~} \mathrm{sEH}{ }^{-/-}$ astrocytes also support the notion that the expression of sEH could suppress the immune responses during astrocyte activation. Similarly, $\mathrm{SEH}^{-/-}$mice that received intraperitoneal injection of LPS showed exacerbated astrocyte activation in the brain, as observed by the elevated expression of glial fibrillary acidic protein (GFAP) and pro-inflammatory markers. Moreover, our data show that the phosphorylation of the signal transducer and activator of transcription 3 (STAT3) was upregulated in activated astrocytes from sEH mouse brains, and the pharmacological blockade of STAT3 activity alleviated the pro-inflammatory effects of sEH deletion in LPS-activated primary astrocytes.

Conclusions: Our results provide evidence, for the first time, showing that sEH negatively regulates astrocytic immune responses and GFAP expression, while the underlying mechanism at least partly involves the downregulation of STAT3 phosphorylation. The discovery of a novel function for $\mathrm{SEH}$ in the negative control of astrocytic immune responses involving STAT3 activation confers further insights into the regulatory machinery of astrocyte activation during the development of neurodegeneration.
\end{abstract}

Keywords: Astrocyte activation, Soluble epoxide hydrolase, Immune responses, GFAP, STAT3

\footnotetext{
* Correspondence: fshie@nhri.org.tw

$\mathrm{CCH}$ and $\mathrm{YHL}$ equally contributed to the manuscript.

${ }^{3}$ Center for Neuropsychiatric Research, National Health Research Institutes,

No.35 Keyan Road, Zhunan Town, Miaoli County 350, Taiwan, Republic of

China

Full list of author information is available at the end of the article
}

(c) The Author(s). 2019 Open Access This article is distributed under the terms of the Creative Commons Attribution 4.0 International License (http://creativecommons.org/licenses/by/4.0/), which permits unrestricted use, distribution, and reproduction in any medium, provided you give appropriate credit to the original author(s) and the source, provide a link to the Creative Commons license, and indicate if changes were made. The Creative Commons Public Domain Dedication waiver (http://creativecommons.org/publicdomain/zero/1.0/) applies to the data made available in this article, unless otherwise stated. 


\section{Introduction}

Astrocytes are major glial cells residing in the brain. Astrocytes become activated during the pathogenesis of many neurodegenerative diseases such as Alzheimer's disease (AD). In $\mathrm{AD}$, activated astrocytes are in proximity to $\beta$-amyloid $(A \beta)$ plaques, one of the pathological hallmarks of $\mathrm{AD}$, which have been implicated in the progression of $\mathrm{AD}[1,2]$. A plethora of effectors derived from activated astrocytes are involved in various physiological functions, including neuronal migration, synaptogenesis, and neuroplasticity, as well as in many pathological conditions [3-5]. Conjointly with the impacts derived from microglial activation, the uncontrolled release of these effectors from activated astrocytes causes neuroinflammation and instigates oxidative stress in the diseased brain [6]. Oxidative stress increases the expression of pro-inflammatory genes, which leads to the increased production of interleukin- 6 (IL-6), tumor necrosis factor $\alpha$ (TNF $\alpha$ ), and prostaglandins, through cyclooxygenase 2 (COX2), and nitric oxide (NO) through the activity of inducible NO synthase (iNOS) [7-9]. The resulting consequences, in turn, release more unwanted effectors that repeatedly propagate oxidative stress, ultimately leading to neurotoxicity. Among the many signaling pathways involved in the induction of pro-inflammatory genes, the phosphorylation of the signal transducer and activator of transcription 3 (STAT3) was recently reported to be critical for the induction of glial fibrillary acidic protein (GFAP) and proinflammatory genes such as TNF $\alpha$ and IL-6 [10-12]. These results suggest that STAT3 may play an important role in the control of astrocyte activation and the associated immune responses.

Glia-mediated neuro-inflammation and subsequent neurodegeneration are common pathological features shared by many neurodegenerative diseases, and the inflammatory responses derived from activated astrocytes exacerbate the pathogenesis of these diseases [13]. However, compelling evidence shows that astrocyte activation can also be beneficial for disease recovery and survival $[14,15]$. Indeed, the ablation of activated astrocytes disrupts glial scar formation, leading to the persistent infiltration of inflammatory cells and the failure to recover the integrity of the blood brain barrier [16, 17]. In $\mathrm{AD}$, it has been shown that activated astrocytes mediate $A \beta$ degradation, suggesting that they may function against $A \beta$ accumulation [18-20]. These findings suggest that astrocyte activation is a double-edged sword that can be both beneficial and detrimental to neuronal functions [21], thus, revealing the regulatory mechanism of astrocyte activation may lead to a better understanding of neuronal survival and death in the diseased brain. The fine-tuning of astrocyte activation that enhances neuronal survival and ablates the self-reinforcing cycles of neuro-inflammation is critical for developing treatments for diseased brain with robust neuro-inflammation [22].

Soluble epoxide hydrolase (sEH) has recently gained increasing attention because the inhibition of hydrolase activity by sEH can result in sustained levels of epoxyeicosatrienoic acids (EETs) and provide neuroprotection in cardiovascular disease and brain injuries [23-28]. Epoxygenases, a subgroup of enzymes in the cytochrome P450 family, metabolize arachidonic acid into hydroxyeicosatetraenoic acids (HETEs) and EETs [29], the latter of which can be further metabolized into less active forms of DiHETEs by sEH, which is encoded by the gene Ephx2. The C-terminus of sEH is the site of the epoxide hydrolysis activity responsible for producing DiHETEs, while the $\mathrm{N}$-terminal domain of sEH possesses phosphatase activity, with lysophosphatidic acid as its endogenous substrate [30]. sEH is primarily found in the liver, the kidney, the cardiovascular system, and the brain [31, 32]. The expression level of sEH was increased in mice with pilocarpineinduced epilepsy, while the genetic knockout of sEH in mice made them more susceptible to inducible seizures [33]. However, the mechanism through which astrocytic $\mathrm{sEH}$ regulates glia-mediated neuroinflammation remains unclear. In this study, we demonstrate that the increased expression of $\mathrm{sEH}$ in activated astrocytes in the vicinity of $\mathrm{A} \beta$ plaques was associated with the progression of $\mathrm{AD}$ in a mouse model. The immune regulatory roles of astrocytic sEH in LPS-activated astrocytes were investigated using in vitro and in vivo models.

\section{Materials and methods Materials}

Lipopolysaccharide (LPS, Escherichia coli O55:B5) was purchased from Calbiochem. Antibodies for inducible nitric oxide synthase (iNOS) and $\beta$-actin were purchased from BD Transduction Lab and Novus Biologicals, respectively. Cyclooxygenase-2 (COX-2) and glial fibrillary acidic protein (GFAP) were purchased from Abcam. Total STAT3 and phospho-STAT3 (p-STAT3) were purchased from Cell Signaling Technology. ELISA kits for TNF $\alpha$ and IL-6 were purchased from Invitrogen. 12-(3adamantan-1-yl-ureido)-dodecanoic acid (AUDA) and $\mathrm{N}$-acetyl-S-farnesyl-L-cysteine (AFC) were purchased from Sigma-Aldrich. Poly-ornithine was purchased from BD Biosciences. Papain and DNase I were purchased from Worthington Biochemical. Fetal bovine serum was purchased from HyClone and heat-inactivated. Culture media and penicillin/streptomycin were purchased from Gibco, and other common chemicals were from SigmaAldrich, unless stated otherwise.

\section{Animals}

Breeding pairs of wild-type (Wt) controls and APPswe/ PSEN1dE9 (APP/PS1) transgenic mice, overexpressing 
both human APP695 Swedish mutations (HuAPP695swe) and a mutant human presenilin 1 (PS1-dE9), were originally obtained from the Jackson laboratory. The transgenic genotypes of both APP and PS1 were detected using PCR, according to the manufacturer's instruction. APP/PS1 transgenic mice that were heterozygous for the transgenes and Wt littermates were used as controls. Given that LPS has been widely used to induce inflammatory responses $[9,12,22]$, a single dose of LPS was administered intraperitoneally to mice at $5 \mathrm{mg} / \mathrm{kg}$ body weight. Wt and $\mathrm{sEH}^{-/-}$ mice that received a single intraperitoneal injection of saline were served as sham control. Forty-eight hours after LPS injection, brain tissues were harvested for later experiments. For primary astrocytic cultures, Wt mice were purchased from the National Laboratory Animal Center (Taipei, Taiwan), and $\mathrm{sEH}^{-/-}$mice (C57BL/6/B6.129X$\left.E p h x 2^{t m 1 G o n z} / \mathrm{J}\right)$ were generously provided by Dr. TzongShyuan Lee. Mice were maintained at the NHRI Laboratory Animal Center with free access to food and water. All experiments were performed as approved by the NHRI IACUC.

\section{Primary astrocytic cultures}

Primary astrocytic cultures were derived from the cortices of P1 to P3 neonates. Cells were dissociated using an enzyme solution, containing Dulbecco's modified Eagle's medium (DMEM), ethylenediamine tetraacetic acid (0.5 $\mathrm{mmol} / \mathrm{L})$, L-cysteine $(0.2 \mathrm{mg} / \mathrm{ml})$, papain $(15 \mathrm{U} / \mathrm{ml})$, and DNase I $(200 \mu \mathrm{g} / \mathrm{ml})$, followed by trituration. Culture medium (DMEM with $10 \%$ fetal bovine serum, $100 \mathrm{U} / \mathrm{ml}$ penicillin, and $100 \mu \mathrm{g} / \mathrm{ml}$ streptomycin) was changed after 24 hours of initial seeding. To obtain enriched primary astrocytes, the astrocytic monolayer was repeatedly trypsinized (at 90\% confluence) and reseeded twice. On the 14th day in vitro (14 DIV), primary astrocytes were reseeded in poly-ornithine-coated 24-well culture plates for experiments, and a fraction of the cells was cultivated on chambered slides at $1 \times 10^{5}$ cells per well, followed by GFAP staining, to determine the purity of the astrocytes. The purity of primary astrocyte cultures was approximately 95\%. Primary cultures were treated with $10 \mathrm{ng} \mathrm{LPS} / \mathrm{ml}$ of culture medium for $24 \mathrm{~h}$. To inhibit STAT3 phosphorylation in LPS-activated astrocytes, 1-h pretreatment with $10 \mu \mathrm{M}$ of the STAT3 inhibitor, stattic (Abcam), was applied.

\section{Genetic manipulations of sEH by using siRNA and over- expression plasmid}

Small interfering RNA duplexes targeting sEH (si-sEH), consisting of a pool of 3 target-specific siRNAs designed to knock down sEH gene expression, and a scramble control were purchased from Santa Cruz Biotechnology, and an Ephx2 mouse cDNA clone for the overexpression of $\mathrm{sEH}$ and a vehicle control were purchased from
OriGene. Primary cell cultures were transfected with siRNAs, plasmid, or controls at a final concentration of $30 \mathrm{nM}$ in serum-free Opti-MEM by using the TransITsiQUEST $^{\mathrm{mm}}$ transfection reagents (Mirus) for $48 \mathrm{~h}$, followed by 24-h treatments of LPS. Quantification of sEH gene expression was evaluated by quantitative polymerase chain reaction (qPCR), as described below.

\section{RNA extraction, reverse-transcription, and qPCR}

Total RNA was extracted with an RNeasy Plus Mini Kit (Qiagen) according to the manufacturer's instructions. RNA $(1 \mu \mathrm{g})$ was reverse transcribed into cDNA using a random primer and a SuperScript III Reverse Transcriptase Kit (Invitrogen). Quantitative PCR was conducted using Luminaris Color HiGreen qPCR Master Mix (Thermo) and an ABI PRISM 7500 Real-Time PCR System. Forward and reverse primer sets for each cDNA were used as follows: 5'-TGGTGGTGACAAGCACATTT-3' and 5'-AAGGCCAAACACAGCATACC-3' (for Nos2, NM_010927.3); 5'-GGCCATGGAGTGGACTTAAA-3' and 5'-CACCTCTCCACCAATGACCT-3' (for COX-2, NM_011198.3); 5'-GAGGGACAACTTTGCACAGG-3' and 5'-TCCTGTCTATACGCAGCCAG-3' (for GFAP, NM_001131020.1); 5'-ATCTCATACCAGGAGAAAGTCAACCT-3' and 5'-TGGGCTCATACCAGGGTTTG-3' (for TNF- $\alpha$, NM_013693.3); 5'-GACCAAGACCATCCAATTCATCTT-3' and 5'-GGAATGTCCACAAACTGATATGCT-3' (for IL-6, NM_031168.1); 5'-TGGT GTGGAACATGGCTCTCT-3' and 5'-ACTGGGATAGATCGGATAACTTTCA-3' (for sEH, NM_007940.4); and $5^{\prime}$-TGTGTCCGTCGTGGATCTGA-3' and 5' GATGCCTGCTTCACCACCTT-3' (for GAPDH, NM_ 008084.3). The average cycle threshold $(\mathrm{Ct})$ value was normalized using the GAPDH signal. Relative transcript levels were calculated as $x=2^{-\Delta \mathrm{Ct}}$, in which $\Delta \mathrm{Ct}=\mathrm{Ct}_{\text {target gene }}{ }^{-}$ $\mathrm{Ct}_{\mathrm{GADH}}$. For cell cultures, each experimental condition was collected from at least 3 independent cultures.

\section{Confocal imaging}

Confocal microscopy was used for semi-quantification and qualitative analysis. Brain samples were subjected to paraformaldehyde ( $4 \%$ in PBS) fixation, overnight, followed by cryoprotection with sucrose (30\% in PBS). Cryosections, at $30 \mu \mathrm{m}$ thick, were subjected to immunohistochemical analysis using antibodies against $A \beta$ (6E10, 1:200, Biolegend), GFAP (astrocyte marker, 1:200, Abcam), p-STAT3 (1:200, Cell Signaling Technology), or sEH (1:100) for overnight incubation at $4{ }^{\circ} \mathrm{C}$. The corresponding secondary antibodies conjugated with Alexa Fluor (Invitrogen) as indicated in the results were applied for $2 \mathrm{~h}$. Tissues were coverslipped with mounting medium (Vector Lab) containing 4,6-diamiino-2-phenylindole (DAPI) for nuclei counterstaining. Images were acquired using a Leica confocal microscopy imaging 
system. GFAP immunoreactivity has been widely used for the evaluation of morphological alterations in activated astrocytes in vivo and thus the quantification of GFAP immunoreactivity was used as a measure of astrocyte activation in this study. Semi-quantification of the immunoreactivity from two sections per mouse was performed using MetaMorph imaging software.

\section{Measurements of sEH activity}

Homogenates of the hippocampus or cortex were subjected to measurements of sEH activity, using an epoxide hydrolase activity assay kit (Cayman Chemistry) according to the manufacturer's instructions. Briefly, the assay utilized Expox Fluor 7, a sensitive fluorescent substrate for sEH that can be used to monitor the activity of sEH. Hydrolysis of the substrate epoxide yielded a highly fluorescent product, 6-methoxy-2-naphthaldehyde, that was monitored at excitation and emission wavelengths of 330 and $465 \mathrm{~nm}$, respectively, using a microplate reader. $\mathrm{sEH}$ activity was presented as $\mathrm{pmol} / \mathrm{min}$ per $\mathrm{mg}$ of protein.

\section{Western blot}

The expression levels of COX-2, iNOS, GFAP, total STAT3, p-STAT3, and $\beta$-actin were determined using cell lysates or brain homogenates. Samples were lysed in lysis buffer $(50 \mathrm{mM}$ Tris, pH 7.4, $150 \mathrm{mM} \mathrm{NaCl}, 0.5 \%$ sodium dodecyl sulfate and protease inhibitor cocktail) and were subjected to electrophoresis, followed by protein transfer onto PVDF membranes and Western blot analyses using antibodies against COX-2 (1:750), iNOS (1:750), GFAP (1:1000), total STAT3 (1:1000), p-STAT3 (1:1000), and $\beta$-actin (1:5000). Targets were detected by the incubation of HRP-labeled secondary antibodies. The corresponding bands, revealed by electrochemiluminescence (ECL) reaction, were analyzed using ImageJ.

\section{Measurements of cytokines}

The culture media from primary astrocytic cultures was subjected to measurements of pro-inflammatory cytokines, including IL- 6 and TNF $\alpha$, using ELISA kits. The assay was performed according to the manufacturers' instructions. Detection of the results was performed using an ELISA plate reader (SpectraMaxM2, Molecular Devices) at a wavelength of $450 \mathrm{~nm}$.

\section{Statistical analysis}

A two-tailed independent Student's $t$ test was used to test significance. For ANOVA, significance for post hoc multiple comparisons between groups was determined with the Bonferroni test using GraphPad Prism software. Data are presented as the mean \pm SEM. Statistical significance was set at $p<0.05$.

\section{Results}

Immunoreactivity of sEH in activated astrocytes increased in parallel with the progression of $A D$

Because activated astrocytes are closely associated with the progression of $\mathrm{AD}$, we first evaluated the expression levels of $\mathrm{sEH}$ in activated astrocytes in the vicinity of $\mathrm{A} \beta$ plaques. Brain tissues from APP/PS1 transgenic mice at different ages, ranging from 5 to 14 months, were used for fluorescent immunohistochemistry (Fig. 1). Because GFAP has been widely used as a marker for astrocyte activation [2], activated astrocytes were identified by immunohistochemistry with anti-GFAP antibody. The results from confocal imaging show that activated astrocytes surrounding $A \beta$ plaques contained very high immunoreactivity for sEH as shown in 14-month-old APP/ PS1 transgenic mice (Fig. 1, upper panel). However, a few astrocytes in the vicinity of $A \beta$ plaques exerting less activated morphology contained little immunoreactivity for sEH. Similarly, GFAP-positive astrocytes with resting morphology in age-matched wild-type (Wt) littermates were negative for $\mathrm{sEH}$. Semi-quantification of the total intensity of $\mathrm{sEH}$ immunoreactivity within clusters of GFAP-positive, activated astrocytes, centered by A $\beta$ plaques with a diameter of $200 \mu \mathrm{m}$, was performed (Fig. 1, lower panel). The data indicate that the levels of $\mathrm{sEH}$ immunoreactivity in the vicinity of $A \beta$ plaques escalated with aging and in parallel with the progression of astrocyte activation in APP/PS1 transgenic mice. As the number of activated astrocytes increased in APP/PS1 transgenic mice compared to the age-matched Wt littermates, the levels of $\mathrm{sEH}$ immunoreactivity also increased. These data suggest that the extent of $\mathrm{sEH}$ immunoreactivity may be related to the activation state of astrocytes and exposure to $A \beta$ may not necessarily trigger the expression of $\mathrm{sEH}$.

\section{Expression of sEH in primary astrocytes regulated the LPS-induced immune response}

To reveal the possible role of astrocytic sEH observed in the activated astrocytes in vivo, we established in vitro models, using LPS to activated primary astrocytes, and then examined the effects of sEH expression on the regulation of astrocyte activation using genetic manipulations. The expression levels of $\mathrm{sEH}$ in primary astrocytes derived from wt mice were genetically manipulated by the RNA silencing of $\mathrm{sEH}$ (si-sEH) and the overexpression of $\mathrm{sEH}$ (over-sEH). The levels of sEH mRNA in si-sEH and oversEH astrocytes were $28 \pm 3 \%$ and $5.8 \pm 1.2 \times 10^{6} \%$ of the controls, respectively, as measured by qPCR (Additional file 1: Figure S1A, B). Unexpectedly, the mRNA levels of $\mathrm{sEH}$ in si-scrambled control astrocytes and nontransfected primary astrocytes appeared to be downregulated by LPS treatments (Additional file 1: Figure S1C). However, sEH activity was slightly reduced in primary 

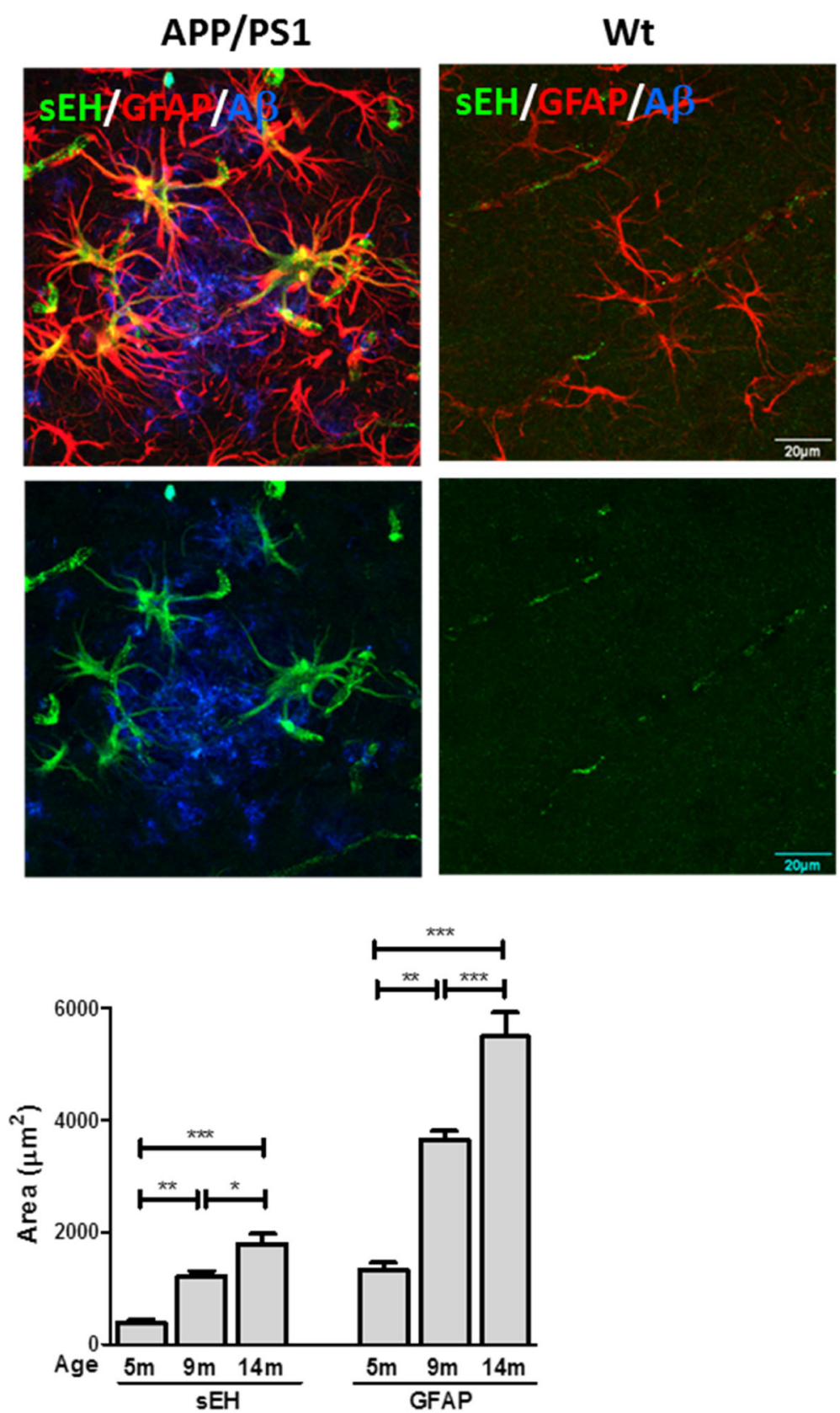

Fig. 1 Increased immunoreactivity of SEH in activated astrocytes occurred in parallel with the progression of AD. Representative confocal images show that sEH immunoreactivity (green) was found within activated astrocytes (red) in the vicinity of A $\beta$ plaques (blue) in APP/PS1 mice at the age of 14 months, whereas GFAP-positive astrocytes with less activated morphology showed minimal immunoreactivity for sEH. In age-matched Wt littermates, GFAP-positive astrocytes were observed with resting morphology and contained little immunoreactivity for sEH. In the right panel, the semi-quantification of the total intensity of $\mathrm{sEH}$ immunoreactivity centered by A $\beta$ plaques with a diameter of $200 \mu \mathrm{m}$ in APP/PS1 mice at 5 months $(5 \mathrm{~m}, n=5), 9$ months $(9 \mathrm{~m}, n=10)$, and 14 months $(14 \mathrm{~m}, n=11)$ of age is shown. The data indicate that the levels of sEH immunoreactivity escalated with aging and in parallel with the progression of astrocyte activation in APP/PS1 transgenic mice. Data are presented as the mean \pm SEM. One-way ANOVA and Bonferroni multiple comparison test were performed. ${ }^{*} p<0.05,{ }^{* *} p<0.01,{ }^{* * *} p<0.001$

astrocytes treated with LPS $(79 \pm 4 \mathrm{pmol} / \mathrm{min} / \mathrm{mg}, n=6)$ compared to non-treated control $(86 \pm 6 \mathrm{pmol} / \mathrm{min} / \mathrm{mg}$, $n=6$ ). After genetic manipulation of $\mathrm{sEH}$ for $24 \mathrm{~h}$, data show that the expression levels of sEH did not affect the immunity of astrocytes at the basal condition (Fig. 2), while the LPS-induced protein expression levels of proinflammatory markers were significantly increased si-sEH astrocytes compared to those in the controls. As indicated in Fig. 2a, LPS-induced expression levels of iNOS and COX-2 in si-sEH astrocytes as measured by Western blot 


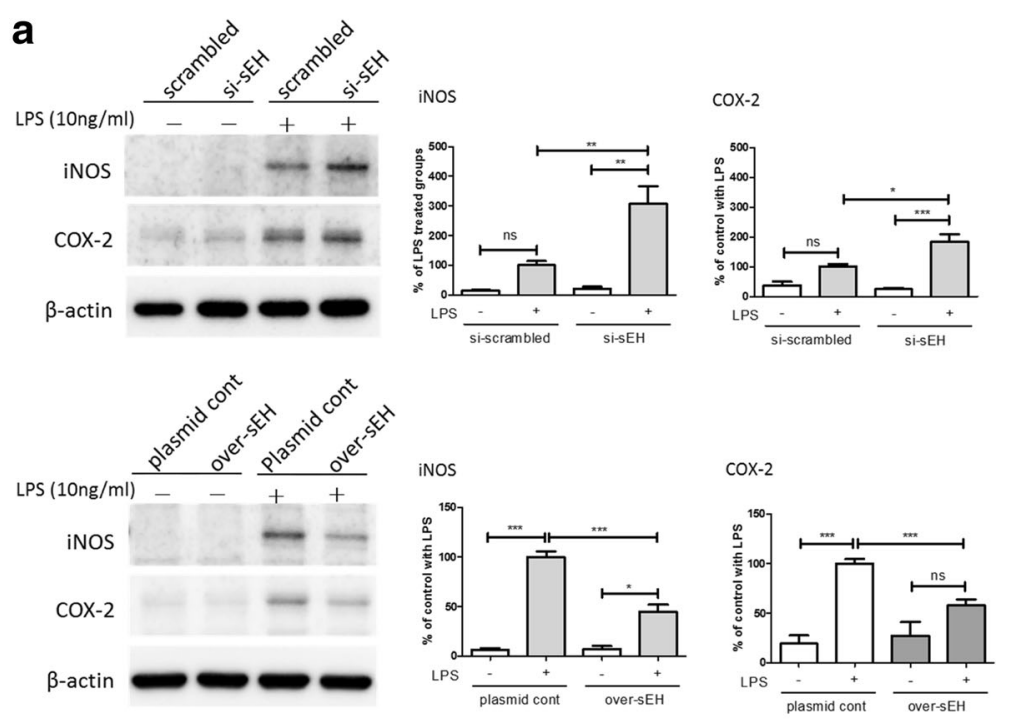

b
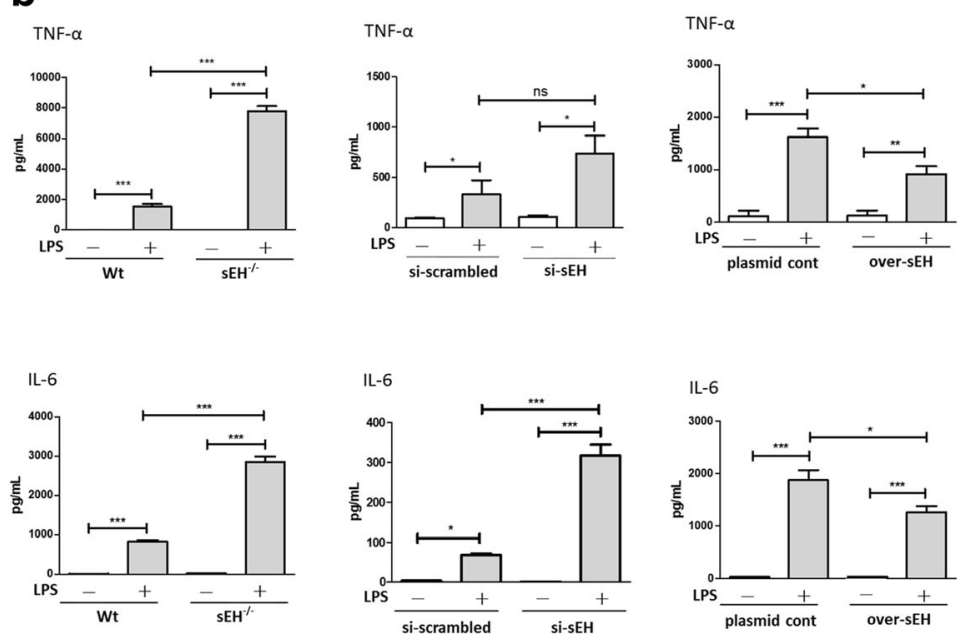

Fig. 2 Expression of astrocytic sEH regulated the LPS-induced expression of pro-inflammatory markers. The LPS-induced protein expression levels of pro-inflammatory markers in si-sEH primary astrocytes $(n=9)$, including iNOS $(p<0.01)$ and COX-2 $(p<0.05)$, were significantly increased compared to those in the controls $(n=9)$, while over-sEH astrocytes $(n=8)$ showed a reduction in LPS-induced expression levels of iNOS $(p<0.001)$ and COX-2 $(p<0.001)(\mathbf{a})$. Similarly, the secretion of LPS-induced TNFa and IL-6 from primary astrocytes derived from sEH ${ }^{-1}$ mice $(n=12)$ was significantly increased ( $p<0.001$ for both) compared to the LPS-treated controls $(n=10)$, while the secretion of TNFa and IL- 6 from over-sEH astrocytes $(n=4)$ was reduced $(p<0.05$ for both) compared to the LPS-treated controls $(n=4)(\mathbf{b})$. In si-sEH astrocytes $(n=4)$, IL-6 secretion was significantly increased $(p<0.001)$ compared to the LPS-treated controls $(n=4)$, while TNFa was slightly increased compared to the LPS-treated controls. Changes in the expression level of sEH had no effects on the basal immunity of astrocytes without the challenge of LPS. Data are presented as the mean \pm SEM. Statistical analysis was performed using one-way ANOVA and Bonferroni multiple comparison test. ${ }^{*} p<0.05,{ }^{* *} p<0.01,{ }^{* * *} p<0.001$

were $307 \pm 59 \%(p<0.01)$ and $183 \pm 25 \%(p<0.05)$ of the LPS-treated controls, respectively. In contrast, over-sEH astrocytes showed a reduction in LPS-induced expression levels of iNOS $(45 \pm 7 \%$ of the LPS-treated controls, $p<$ $0.001)$ and COX-2 (58 $\pm 6 \%$ of the LPS-treated controls, $p<0.001)$. Although levels of iNOS and COX-2 were not affected in astrocytes derived from $\mathrm{sEH}$ knockout $\left(\mathrm{sEH}^{-/-}\right)$ mice (Additional file 1: Figure S2), the secretion of LPSinduced TNFo $(7773 \pm 333 \mathrm{pg} / \mathrm{ml}, p<0.001)$ and IL-6
$(2853 \pm 141 \mathrm{pg} / \mathrm{ml}, p<0.001)$ in $\mathrm{sEH}^{-/-}$astrocytes as measured by ELISA was significantly increased compared to the LPS-treated controls $(1523 \pm 168 \mathrm{pg} / \mathrm{ml}$ and $828 \pm 44$ $\mathrm{pg} / \mathrm{ml}$ for TNF $\alpha$ and IL6, respectively). TNF $\alpha$ (918 \pm 157 $\mathrm{pg} / \mathrm{ml}, p<0.05)$ and IL-6 $(1268 \pm 115 \mathrm{pg} / \mathrm{ml}, p<0.05)$ secretion in over-sEH astrocytes was reduced compared to the LPS-treated controls $(1620 \pm 164 \mathrm{pg} / \mathrm{ml}$ and $1876 \pm$ $195 \mathrm{pg} / \mathrm{ml}$ for TNF $\alpha$ and IL6, respectively) (Fig. 2b). In sisEH astrocytes, IL-6 $(317 \pm 27 \mathrm{pg} / \mathrm{ml}, p<0.001)$ secretion 
was significantly increased compared to the LPS-treated controls $(68 \pm 4 \mathrm{pg} / \mathrm{ml})$, while TNF $\alpha(740 \pm 176 \mathrm{pg} / \mathrm{ml})$ was slightly increased compared to the LPS-treated controls $(332 \pm 141 \mathrm{pg} / \mathrm{ml})$.

The effects of $\mathrm{sEH}$ expression on the mRNA levels of the pro-inflammatory markers were then evaluated by qPCR. Similar to that observed at protein levels, the mRNA levels of the pro-inflammatory markers, including iNOS, COX-2, IL-6, and TNF $\alpha$, were higher in si-sEH or $\mathrm{sEH}^{-/-}$primary astrocytes than those in the respective controls as shown in Fig. 3 a and b. However, the mRNA levels of the pro-inflammatory markers were slightly reduced in over-sEH astrocytes. Of note, LPS treatment increased the mRNA levels of iNOS and COX-2 in the controls, but the mRNA levels of IL- 6 and TNF $\alpha$ were not changed. The mRNA levels of pro-inflammatory cytokines induced by LPS in primary astrocytes were only slightly increased, probably due to the use of a low dose of LPS at $10 \mathrm{ng} / \mathrm{ml}$. However, in primary astrocyte cultures, the secretion of IL- 6 and TNFa induced by low dose of LPS continued to accumulate, resulting in a significant increase of cytokine secretion as shown in Fig. 2b. These data suggest that astrocytic sEH could act as a suppressor in the regulation of the LPS-activated immune response at both the protein and mRNA levels, and the absence of astrocytic sEH could escalate the immune response. However, the overexpression of astrocytic sEH appears to attenuate the pro-inflammatory markers only at the protein level. Thus, gene expression may not contribute to the anti-inflammatory effects of sEH overexpression, and the roles of $\mathrm{sEH}$ in the regulation of astrocyte activation at either the mRNA or protein level might be involved in multiple regulatory pathways.

\section{The LPS-induced astrocyte activation was exacerbated in the brains of $\mathrm{sEH}^{-/-}$mice}

The effect of sEH in the negative regulation of astrocyte activation was further tested using animal models, with cerebral inflammation induced by the peripheral delivery of LPS. Our data show that intraperitoneal injections of LPS $(5 \mathrm{mg} /$ $\mathrm{kg}$ body weight) in $\mathrm{sEH}^{-/-}$mice exacerbated astrocyte activation in the brain as evidenced by the enhanced reactive morphology of GFAP-positive astrocytes at $48 \mathrm{~h}$ after the treatments (Fig. 4a). By Western blot analysis, the protein expressions of GFAP in the cortex $(150 \pm 19 \%$ of Wt control with LPS, $p<0.05)$ and hippocampus $(174 \pm 22 \%$ of Wt control with LPS, $p<0.05)$ were increased in LPS-treated $\mathrm{sEH}^{-1-}$ mice compared to those in the Wt counterparts (Fig. 4b). In mice receiving saline injection, there was no significant difference in the protein levels of GFAP between Wt $(100 \pm 11 \%$ and $100 \pm 18 \%$ for cortex and hippocampus, respectively, $n=5)$ and $\mathrm{sEH}^{-/-}$mice $(84 \pm 13 \%$ and $88 \pm 15 \%$ of Wt control with saline for cortex and hippocampus, respectively, $n=4)$. An increased hippocampal iNOS at protein levels was also found in $\mathrm{sEH}^{-/-}$mice that received LPS (146 $\pm 7 \%$ of Wt control with LPS, $p<0.05$ ), whereas cortex iNOS was slightly increased.

The extent of astrocyte activation was then measured by the expressions at mRNA levels for GFAP and proinflammatory markers, including iNOS, COX-2, IL-6, and TNFo in the hippocampus and cortex. Data indicate that intraperitoneal injections of LPS slightly elevated those genes as compared to saline controls, some of which were significantly increased in $\mathrm{sEH}^{-/-}$mice. One explanation for the slight increase of these inflammatory markers induced by LPS might be due to the time-dependent effects of LPS on mRNA expression. As shown in Fig. 5, the LPStreated $\mathrm{sEH}^{-1-}$ mice expressed higher mRNA levels of iNOS $(264 \pm 73 \%$ of the LPS-treated Wt control, $p<0.01)$, IL-6 ( $340 \pm 119 \%$ of the LPS-treated Wt control, $p<0.01)$, and TNFa $(450 \pm 121 \%$ of the LPS-treated Wt control, $p<0.001)$ in the hippocampus than the LPS-treated Wt mice. However, mRNA levels of GFAP and COX-2 in the hippocampus were slightly increased in the LPS-treated $\mathrm{sEH}^{-/-}$mice as compared to the LPS-treated wt mice. In the cortex, the LPS-treated $\mathrm{sEH}^{-/-}$mice expressed higher mRNA levels of COX-2 (198 $\pm 48 \%$ of the LPS-treated Wt control, $p<0.05)$ and IL-6 (263 $\pm 89 \%$ of the LPS-treated Wt control, $p<0.05)$ than the LPS-treated wt mice, whereas mRNA levels of GFAP, iNOS, and TNF $\alpha$ were slightly increased. These results support the findings of our in vitro study, suggesting that sEH may function as a suppressor in the regulation of the LPS-activated immune response in astrocytes, while the genetic deletion of $\mathrm{sEH}$ may exacerbate astrocyte activation and the associated immune response. However, the levels of GFAP and the proinflammatory markers at the basal conditions were not affected by the genetic deletion of sEH. These findings suggest that the functional effects of $\mathrm{sEH}$ on the regulation of the astrocytic immune response might be associated with the activation status, and it is conceivable to speculate that the LPS-activated signaling pathways may be involved in the regulatory function of $\mathrm{sEH}$ during astrocyte activation. In line with this speculation, our data indeed showed that $\mathrm{sEH}$ activity in the hippocampus and cortex was significantly suppressed in the brains of mice that received intraperitoneal injections of LPS (Fig. 5c). These findings also echo our data as described above that mRNA levels of $\mathrm{sEH}$ were suppressed in primary astrocytes treated with LPS, although the reduction of sEH by acute activation by LPS is contrary to the increase of astrocytic sEH in aged APP/PS1 mice with chronic astrogliosis.

\section{Regulation of astrocyte activation by $\mathrm{sEH}$ involves the suppression of STAT3 phosphorylation}

To explore the signaling pathways that could be attributed to the negative regulatory role of $\mathrm{SEH}$ in the LPSinduced astrocytic immune response, we examined the 
a
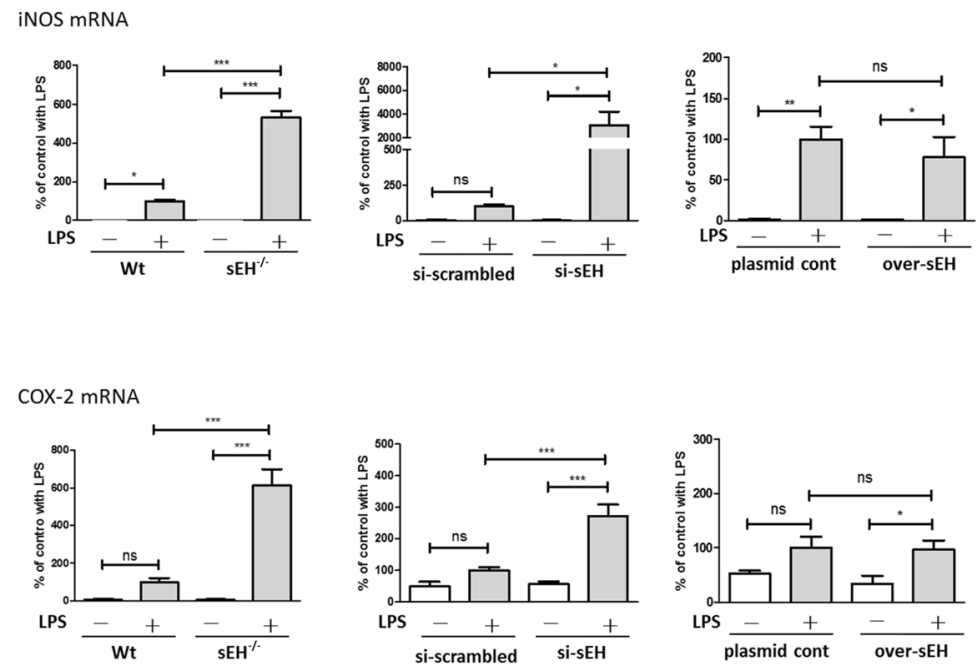

b
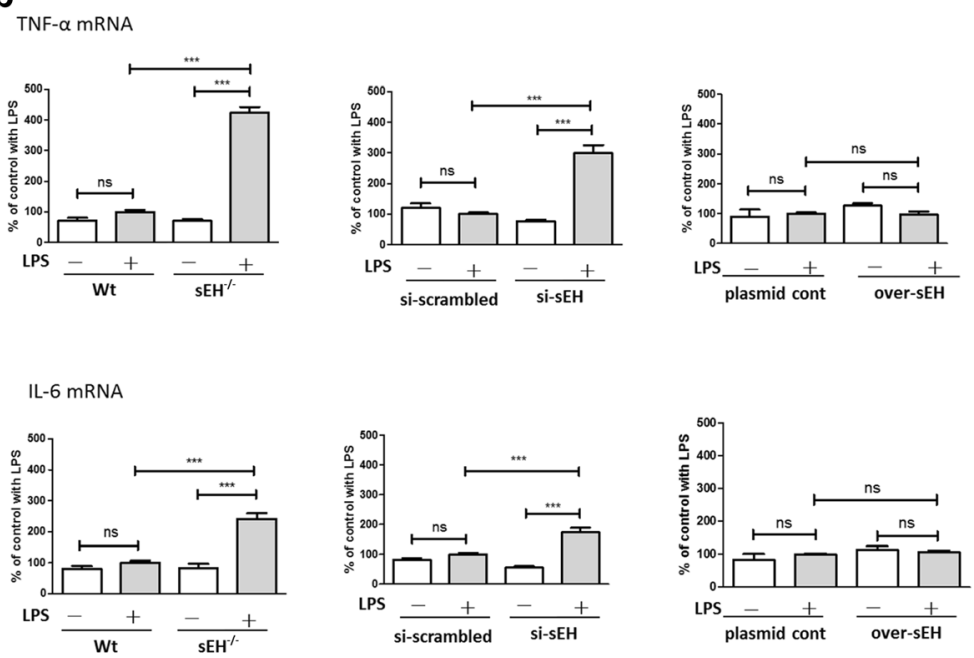

Fig. 3 The effects of sEH expression in primary astrocytes on the mRNA levels of LPS-induced pro-inflammatory markers. LPS activated primary astrocytes, as evidenced by significant increases in the mRNA levels of iNOS and COX-2 in Wt, si-scrambled, and plasmid controls (a). Manipulations of astrocytic sEH levels using si-sEH $(n=9) \mathrm{or} \mathrm{sEH}^{-/-}(n=6)$ led to further increases in iNOS $\left(p<0.05\right.$ for si-sEH and $p<0.001$ for sEH $\left.{ }^{-/-}\right)$ and COX-2 ( $p<0.001$ for both) mRNA levels, but the mRNA levels were slightly reduced in over-sEH $(n=5)$. Similarly, the mRNA levels of TNFa and IL-6 in primary astrocytes with either si-sEH or sEH ${ }^{-1-}$ were increased ( $p<0.001$ for both) compared to respective controls, and over-sEH had no effects on the mRNA levels $(\mathbf{b})$. Data are presented as the mean \pm SEM. One-way ANOVA and Bonferroni multiple comparison test were performed. ${ }^{*} p<0.05$; ${ }^{* *} p<0.01 ;{ }^{* *} p<0.001$

activation of nuclear factor kappa B (NFkB), P38, and janus kinase 2 (JAK2)/STAT3 in $\mathrm{sEH}^{-/-}$and $\mathrm{Wt}$ mouse brains. The data from the Western blot analysis show that intraperitoneal injections of LPS upregulated the phosphorylation of STAT3 in mouse brains (Fig. 6a), but not JAK2, NFkB, or P38 (data not shown). Intriguingly, the phosphorylation of STAT3 was significantly higher in the cortex of $\mathrm{sEH}^{-1-}$ mice that received LPS (302 $\pm 56 \%$ of Wt control, $p<0.05$ ) than in Wt counterparts $(161 \pm 13 \%$ of Wt control). Although a slight elevation was found in the hippocampus of $\mathrm{sEH}^{-/-}$mice $(541 \pm 42 \%$ of Wt control) compared to the control that received LPS $(387 \pm 19 \%$ of Wt control), phosphorylation of STAT3 in the hippocampus of LPS-treated $\mathrm{sEH}^{-/-}$mice was significantly increased $(p<0.05)$ in the hilus of the dentate gyrus as demonstrated by confocal imaging with quantification (Fig. 6b). The results show that $\mathrm{sEH}^{-/-}$mice that received intraperitoneal injection of LPS showed higher levels of STAT3 phosphorylation than Wt counterparts, whereas the immunoreactivity of phosphorylated STAT3 was completely co-localized within GFAP-positive astrocytes. Based on these findings, we speculate that $\mathrm{sEH}$ may function as a negative 

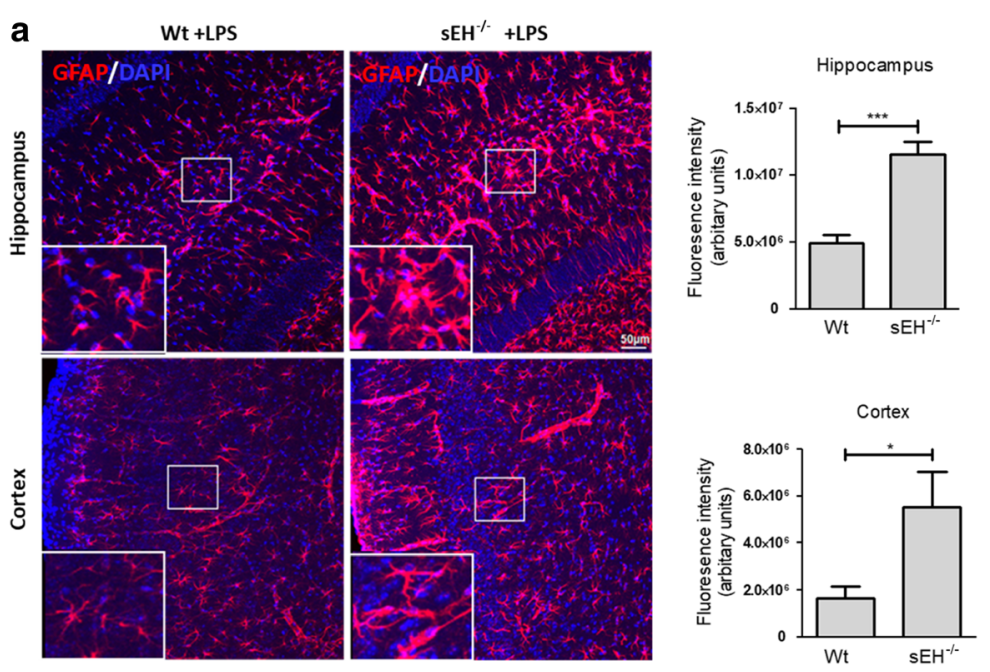

b
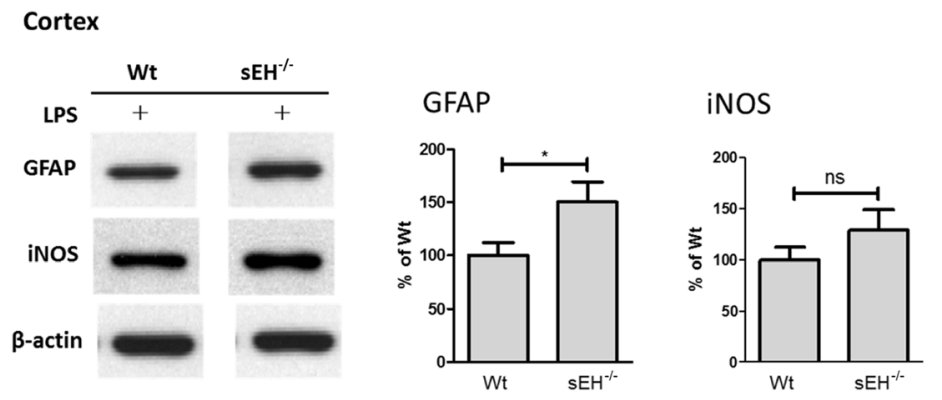

Hippocampus
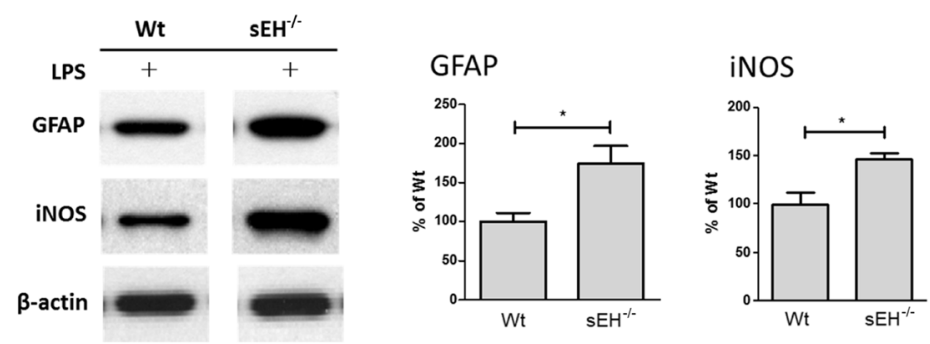

Fig. 4 Astrocyte activation was exacerbated in the brains of $\mathrm{sEH}^{-/-}$mice treated with LPS. Confocal imaging shows that astrocyte activation was exacerbated in $\mathrm{SEH}^{-/}$mice that received intraperitoneal injections of LPS compared to Wt counterparts, as evidenced by the enlarged morphology of GFAP-positive astrocytes in both the cortex and hippocampus (a). A magnified image of the boxed area is presented at the lower left of each image for a closer look of GFAP immunoreactivity. This notion is supported by the semi-quantification data, showing that the total intensity of GFAP immunoreactivity is increased in $\mathrm{SEH}^{-/-}$mice with LPS $(n=5, p<0.05$ for cortex and $p<0.001$ for hippocampus) compared to Wt counterparts $(n=6)$. Similarly, Western blot analysis also shows increased protein expressions of GFAP and iNOS in both the cortex and hippocampus of $\mathrm{sEH}^{-/-}$mice that received intraperitoneal injections of LPS compared to Wt counterparts, although changes of cortical

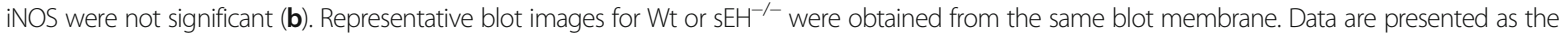
mean \pm SEM. Statistical analysis was performed using Student's $t$ test and significance is indicated. ${ }^{*} p<0.05 ;{ }^{*} p<0.01$

regulator of astrocyte activation involving the suppression of STAT3 phosphorylation.

We then examined the effects of a pharmacological blockade of STAT3 activity on the expression levels of pro-inflammatory markers in primary astrocytes treated with si-sEH. Similar to what was observed in Fig. 3, the levels of LPS-induced TNF $\alpha$ (3736 $\pm 136 \%$ of the scrambled control, $p<0.001)$ and IL-6 $(1371 \pm 38 \%$ of the scrambled control, $p<0.001)$ mRNA were higher in si$\mathrm{sEH}$ astrocytes than those in the scrambled control astrocytes $(1258 \pm 33 \%$ and $392 \pm 24 \%$ of the scrambled control for TNF $\alpha$ and IL-6, respectively) (Fig. 7a). The inhibition of STAT3 phosphorylation by the pretreatment of stattic, a STAT3 inhibitor, significantly attenuated the LPS-induction of TNF $\alpha$ mRNA in both si$\mathrm{sEH}$ astrocytes and the scrambled controls. Intriguingly, 
Hung et al. Journal of Neuroinflammation

(2019) $16: 123$

Page 10 of 16

a

Hippocampus
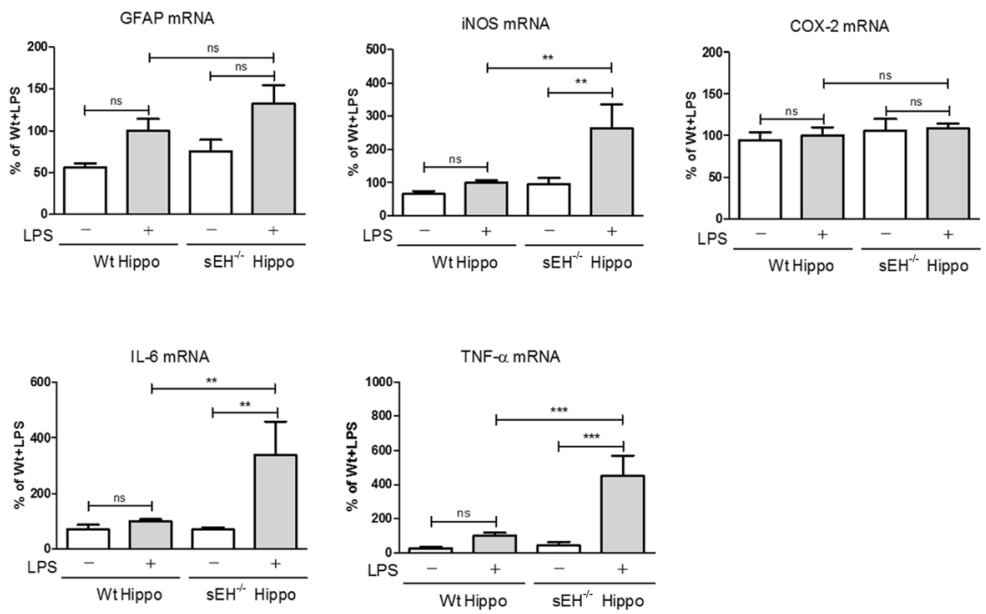

b cortex
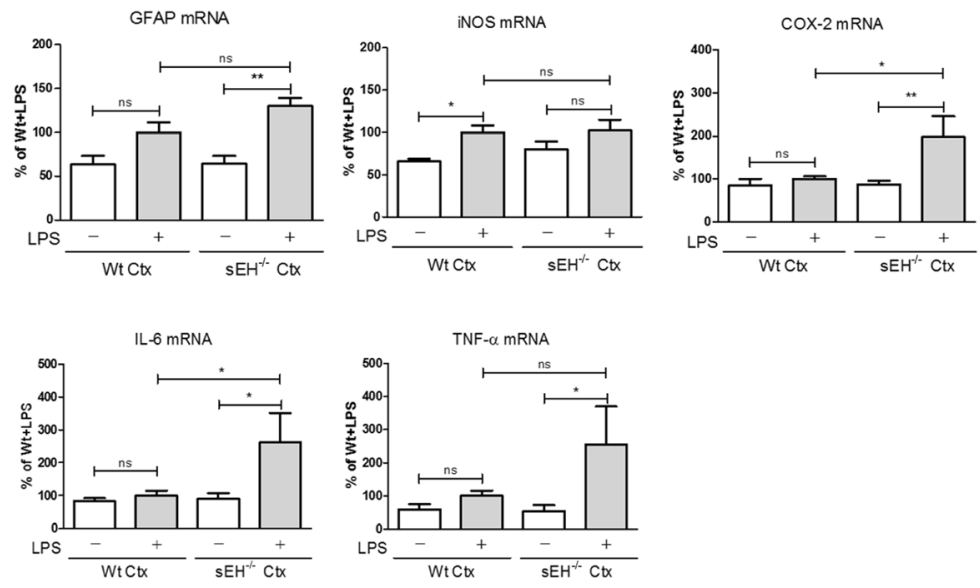

C Activity of SEH
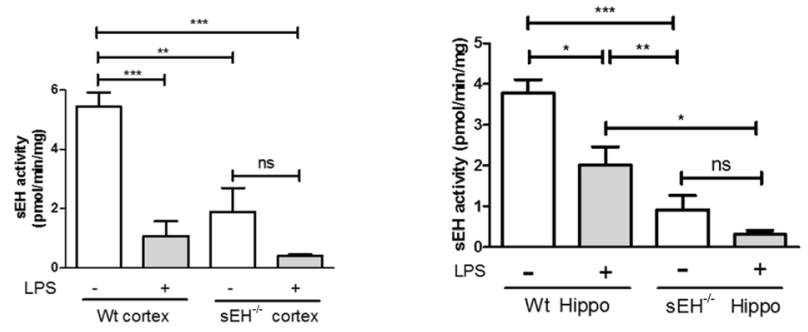

Fig. 5 (See legend on next page.) 
(See figure on previous page.)

Fig. 5 The LPS-induced mRNA expressions of pro-inflammatory markers were enhanced in the brains of $\mathrm{sEH}^{-1-}$ mice. After intraperitoneal injections of LPS, the mRNA levels of GFAP and pro-inflammatory markers, including iNOS, COX-2, IL-6, and TNFa were evaluated in the hippocampus (a) and cortex (b) by qPCR. The data indicate that the basal levels of mRNA for all markers were comparable between wt $(n=9)$ and sEH ${ }^{-1-}(n=8)$ mice that received saline treatments. LPS treatments in Wt $(n=10)$ slightly increased the mRNA expression levels of GFAP and the pro-inflammatory markers in the examined brain regions except for iNOS in the cortex $(p<0.05)$, whereas LPS treatments in sEH ${ }^{-1-}$ mice $(n=6)$ significantly increased iNOS $(p<$ $0.01)$, IL-6 ( $p<0.01)$, and TNFa $(p<0.001)$ in the hippocampus and GFAP $(p<0.01), \operatorname{COX}-2(p<0.05), \mathrm{IL}-6(p<0.05)$, and TNFa $(p<0.05)$ in the cortex. Compared to Wt control with LPS, the mRNA levels of iNOS $(p<0.01)$, IL-6 $(p<0.01)$, and TNFa $(p<0.001)$ in the hippocampus were further enhanced in sEH ${ }^{-1-}$ mice that received LPS. Similarly, significant increases of COX-2 $(p<0.05)$ and IL-6 $(p<0.05)$ in the cortex of sEH ${ }^{-/-}$mice with LPS were found. These data suggest that LPS treatments may result in higher mRNA expressions of some pro-inflammatory markers in the brain of sEH ${ }^{-/}$mice than those in Wt. c The activity of sEH in the cortex and the hippocampus was lower in sEH ${ }^{-1-}$ mice $(n=4, p<0.001)$ than in wt mice $(n=5)$. A significant suppression of sEH activity was found in the hippocampus $(p<0.05)$ and cortex $(p<0.001)$ of Wt mice with LPS $(n=6)$. The activity of sEH in $\mathrm{SEH}^{-/-}$mice with LPS $(n=5)$ was slightly reduced, but not significant. Data are presented as the mean \pm SEM. One-way ANOVA and Bonferroni multiple comparison test were performed. ${ }^{*} p<0.05 ;{ }^{* *} p<0.01 ;{ }^{* *} p<0.001$.

the inhibition of STAT3 phosphorylation suppressed the LPS-induction of IL-6 mRNA in si-sEH astrocytes, but not in the scrambled controls. These data suggest that, in the presence of $\mathrm{sEH}$, the LPS-activated upregulation of TNF $\alpha$ mRNA in astrocytes may act at least partly through a mechanism involving STAT3 phosphorylation, whereas induction of IL- 6 mRNA by LPS may not require STAT3 phosphorylation. Importantly, in the absence of sEH, further upregulation of TNF $\alpha$ and IL-6 mRNA may require STAT3 phosphorylation. Therefore, sEH may function as a negative regulator of astrocyte activation and STAT3 phosphorylation may be involved in this regulatory process. Intriguingly, treatments with stattic alone in the scrambled control astrocytes promoted the expressions of these pro-inflammatory cytokines, suggesting that STAT3 activity may exert multiple functions in the regulation of astrocyte immune response.

It has been reported that $\mathrm{sEH}$ possesses phosphatase and hydrolase activities. We then investigated whether the regulation of STAT3 phosphorylation during astrocyte activation was differentially affected by these two functional domains of sEH. Pretreatments of AUDA and AFC were applied, to inhibit the $\mathrm{sEH}$ hydrolase activity and phosphatase activity, respectively, in LPS-activated astrocytes. In Fig. 7b, the data show that STAT3 phosphorylation was upregulated in LPS-activated astrocytes and was further enhanced by pretreatments of AFC $(138 \pm 6 \%$ and $141 \pm 4 \%$ of the control with LPS for $1 \mu \mathrm{M}$ and $10 \mu \mathrm{M}$ of $\mathrm{AFC}$, respectively) but not by pretreatments of AUDA. In contrast, LPS-induced STAT3 phosphorylation was reduced in astrocytes overexpressing sEH $(59 \pm 5 \%$ of the control with LPS, $p<0.05)$ compared to the control with LPS (100 $\pm 5 \%)$ (Fig. 7c). These data suggest that the phosphatase, but not the hydrolase, activity of sEH may contribute to the negative regulation of STAT3 phosphorylation.

\section{Discussion}

Astrocyte activation is an important pathological feature in $\mathrm{AD}$ and many other neurodegenerative diseases [34,
35]. Understanding the underlying regulatory mechanism may confer a molecular basis for the development disease therapies. Our data show that the expression of $\mathrm{sEH}$ was positively associated with the progression of astrocyte activation in APP/PS1 transgenic mice, while sEH immunoreactivity was barely detectable in resting astrocytes with a quiescent phenotype. Importantly, we demonstrated, for the first time, that sEH plays a role in the negative regulation of LPS-induced astrocyte immunity, as evidenced by data from in vivo and in vitro models. Data suggest that the genetic deletion of sEH, either $\mathrm{sEH}^{-1-}$ or si-sEH, enhanced the production of LPS-induced pro-inflammatory markers, whereas the overexpression of $\mathrm{sEH}$ ameliorated the immune response. However, the involvement of microglial activation in the enhanced pro-inflammatory markers found in $\mathrm{sEH}^{-/-}$mice cannot be excluded due to the constant interactions between microglia and astrocytes in the brain. Furthermore, our findings identify sEH as a novel inhibitory effector in the regulation of STAT3 activity and demonstrate the critical role of STAT3 in astrocyte activation. Based on these findings, we propose that the immune-suppressing function of sEH involving STAT3 inhibition may play an important role in the regulation of astrocyte activation.

\section{The expression of $s E H$ in astrocyte activation in $A D$ pathogenesis}

The number of GFAP-positive astrocytes near $A \beta$ plaques increases as AD progresses. Similarly, our data show that astrocytic sEH immunoreactivity increased in parallel with the extent of astrogliosis and was limited to the activated astrocytes in proximity to $A \beta$ plaques. However, astrocytes in the brain of AD mice appear to express different levels of $\mathrm{sEH}$, and less activated astrocytes that were close to $A \beta$ plaques expressed little or no $\mathrm{sEH}$, as shown in the abovementioned results. One possible explanation for these findings is that the different levels of sEH expression in response to an inflammatory microenvironment may be due to the heterogeneity 

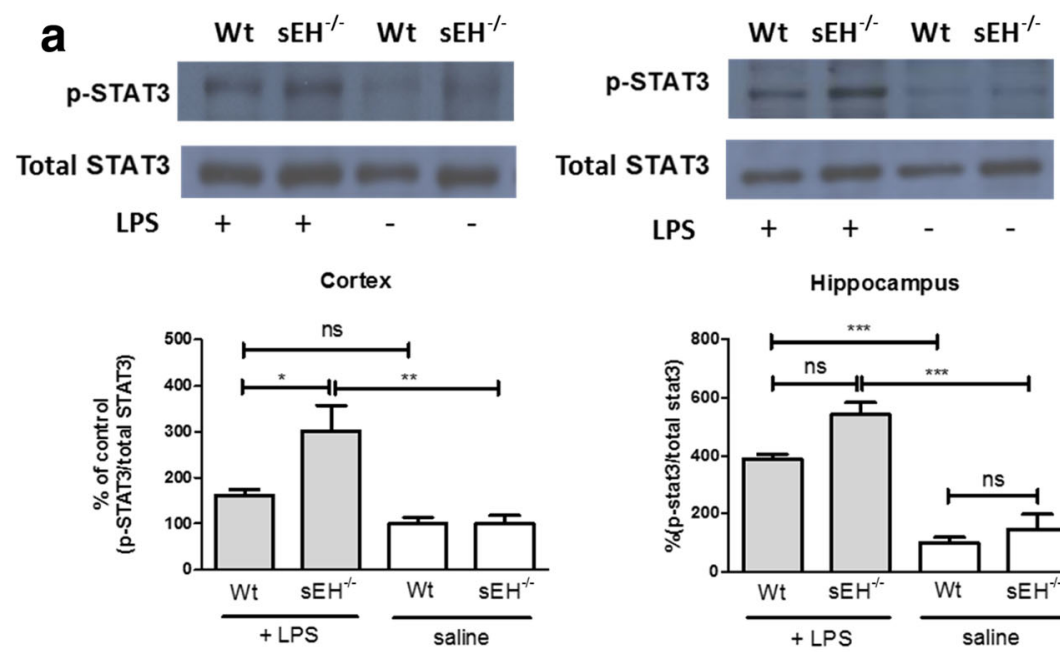

Total STAT3

LPS

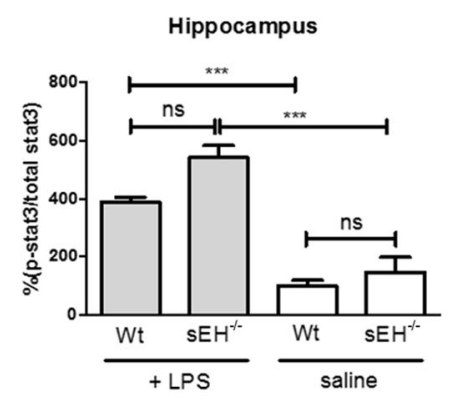

b
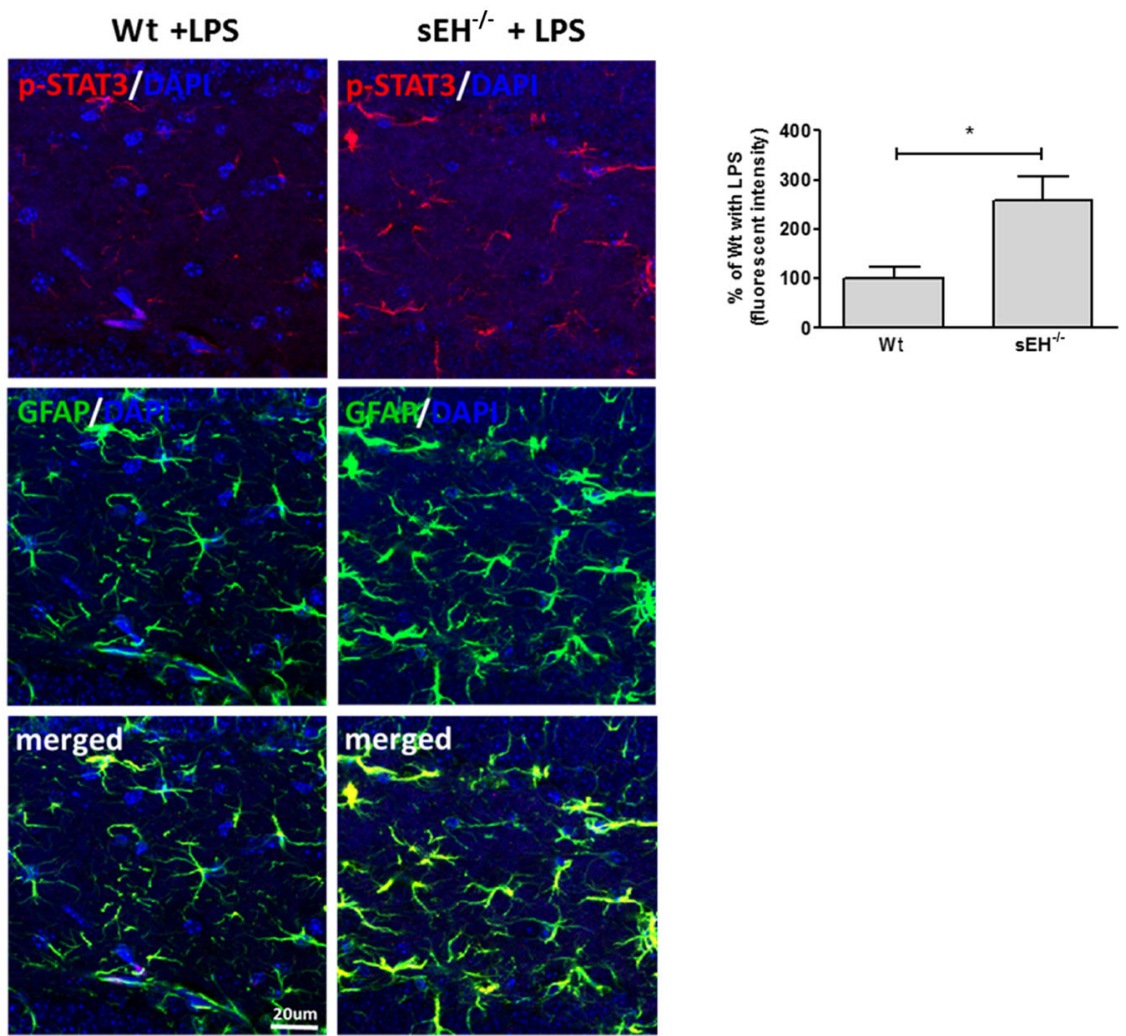

Fig. 6 STAT3 phosphorylation was upregulated in activated astrocytes in $\mathrm{SEH}^{-/-}$mice. Western blot analysis showed that the intraperitoneal injection of LPS significantly upregulated the phosphorylation of STAT3 in the hippocampus of Wt mouse brains $(n=4, p<0.001)$, and this upregulation was also found in the cortex $(p<0.01)$ and the hippocampus $(p<0.001)$ of sEH ${ }^{-/-}$mice $(n=4)(\mathbf{a})$. Intriguingly, LPS-induced phosphorylation of STAT3 was higher in the cortex of sEH ${ }^{-1-}$ mice $(p<0.05)$ than in that of Wt with LPS $(n=4)$. Although the phosphorylation of STAT3 in the hippocampus of $\mathrm{SEH}^{-/-}$mice was slightly upregulated, confocal imaging showed that the phosphorylation of STAT3 (p-STAT3) waS significantly higher $(p<0.05)$ in the hilus of the hippocampus of $\mathrm{sEH}^{-1-}$ mice that received LPS than in Wt counterparts (b). A complete colocalization between phosphorylated STAT3 and GFAP is demonstrated by merged images. P-STAT3 was detected by an antibody against STAT3 phosphorylated at tyrosine 705 (pTyr705). DAPI was used to label nuclei. One-way ANOVA and Bonferroni multiple comparison test were performed (a) and a two-tailed independent Student's t-test was performed (b). ${ }^{*} p<0.05,{ }^{* *} p<0.01,{ }^{* * *} p<0.001$ 
a

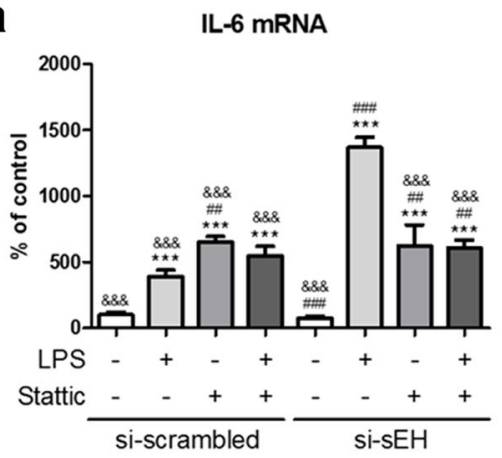

b

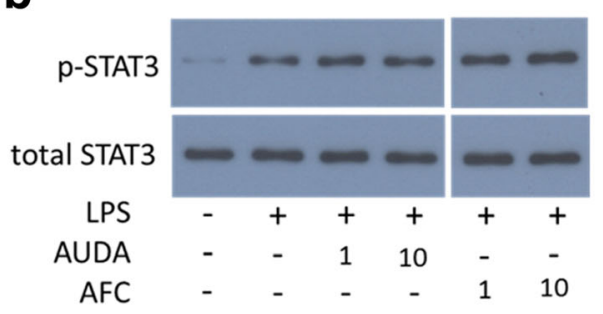

TNF- $\alpha$ mRNA
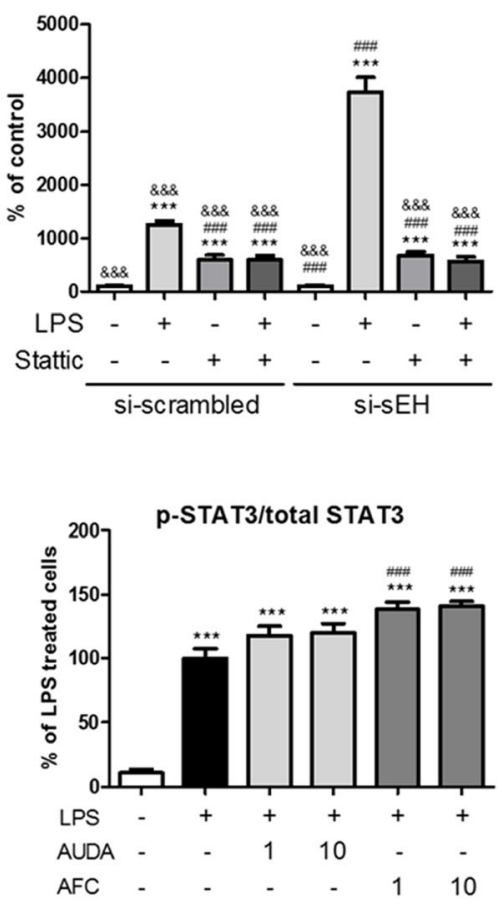

C

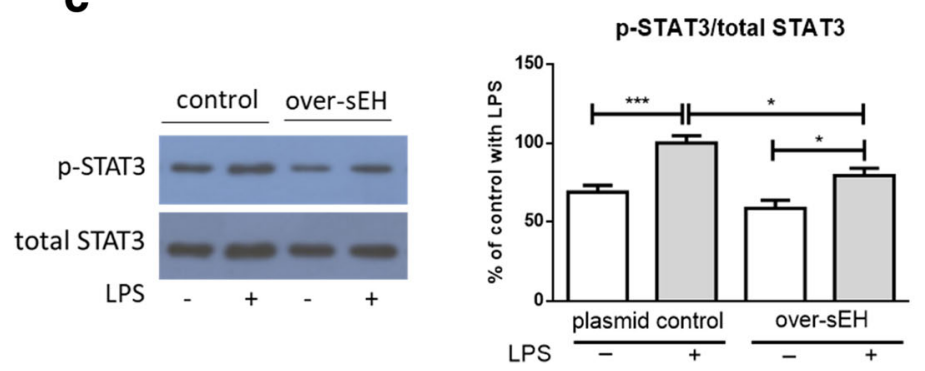

Fig. 7 a-cThe increase of LPS-induced pro-inflammatory markers in si-sEH astrocytes was suppressed by inhibition of STAT3 phosphorylation. The inhibition of STAT3 phosphorylation in LPS-activated astrocytes by the pretreatment of a STAT3 inhibitor, stattic, (10 $\mu$ M) attenuated the enhanced mRNA expression levels of IL-6 and TNFa resulting from si-sEH, as measured by $\mathrm{qPCR}(n=4, p<0.001$ for both IL-6 and TNFa). Stattic significantly suppressed LPS-induced TNFa expression in the scrambled control $(p<0.001)$ while slightly increasing LPS-induced IL-6 expression. Of note, stattic increased the mRNA expression levels for IL-6 $(p<0.001)$ and TNFa $(p<0.001)$ in astrocytes in basal conditions. ${ }^{* * *} p<0.001$, compared to si-sEH control; ${ }^{\#} p<0.01$, \#\# $p<0.001$, compared to si-sEH with LPS; \&\&\& $p<0.001$, compared to si-sEH with LPS. According to the data from Western blot analysis, STAT3 phosphorylation was upregulated in LPS-activated astrocytes $(n=8, p<0.001)$ and was further enhanced by pretreatments with AFC, an sEH phosphatase activity inhibitor, (138 \pm 6 and $141 \pm 4 \%$ of LPS-activated astrocytes for doses at 1 and $10 \mu M$, respectively, $p<0.001)$, but not by pretreatments with AUDA, an sEH hydrolase activity inhibitor, (118 7 and $120 \pm 8 \%$ of LPS-activated astrocytes for doses at 1 and $10 \mu \mathrm{M}$, respectively) (b). All lanes in the representative blot images of the same target protein were obtained from the same blot membrane. ${ }^{* *} p<0.001$, compared to control; \#\# <0.001, compared to control with LPS. In Fig. 7c, LPS-induced STAT3 phosphorylation was reduced in astrocytes overexpressing sEH $(59 \pm 5 \%$ of the control with LPS, $\mathrm{n}=10, p<0.05)$ as compared to that in plasmid control with LPS (100 \pm 5$)$. ${ }^{*} p<0.05,{ }^{* *} p<0.001$. Data are presented as the mean \pm SEM. One-way ANOVA and Bonferroni multiple comparison test were performed

of astrocytes [36]. Indeed, it has been reported that subtypes of activated astrocytes, with distinct functionality in the diseased brain, exist [15]. Alternatively, the increase of $\mathrm{sEH}$ expression in astrocytes may be associated with the progression of chronic disease status, and $A \beta$ exposure per se may not necessarily instigate the expression of $\mathrm{sEH}$ in activated astrocytes. Given that various soluble $A \beta$ species, including $A \beta$ oligomers, are widespread in the brain, the exposure of activated astrocytes to $A \beta$ is inevitable. Moreover, as demonstrated in our data, acute stimulation by LPS reduced, rather than increased, $\mathrm{sEH}$ in mice and primary astrocytes. One explanation is that the unspecified LPS-activated signaling might be involved in the acute effect of LPS on reducing the expression of $\mathrm{sEH}$, whereas chronic activation leading to severe astrogliosis triggers upregulation of astrocytic $\mathrm{sEH}$ to counteract the 
inflammatory state. Therefore, the more the astrocytes are in an advanced and chronic activation, the more sEH is expressed. This speculation echoes our observation showing that intensive sEH immunoreactivity was commonly found within the enlarged processes of highly activated astrocytes in aged APP/PS1 mice. In contrast, activated astrocytes in younger APP/PS1 mice showed less sEH immunoreactivity. Whether the extent of sEH expression in activated astrocytes could be indicative of disease progression and perhaps serves as a biomarker for AD remains to be explored.

Nevertheless, the fact that levels of sEH modulate immune response in activated astrocytes may have significant impacts on $\mathrm{AD}$ pathogenesis. We speculate that high sEH-expressing astrocytes could be a subtype of astrocytes that are attempting to cope with inflammation because the expression of $\mathrm{sEH}$ could be antiinflammatory in activated astrocytes. Therefore, it is conceivable to propose that activated astrocytes with high expression levels of sEH could be beneficial for counteracting the over-activation of astrocyte. However, $\mathrm{sEH}$-overexpressing astrocytes, on the other hand, may reduce their beneficial functions of immune response against the disease progression. In fact, the beneficial functions of activated astrocytes have been recently reported, showing that $A \beta$ plaques with little or no activated astrocytes were associated with dementia status in AD patients [37, 38]. Therefore, the possible adverse effects of $\mathrm{sEH}$ overexpression on over-reducing astrocytic functions remained to be elucidated.

\section{Regulation of astrocyte activation by sEH involving STAT3 inhibition}

Our study support the notion that STAT3 activation can be pro-inflammatory and is involved in triggering the expression of pro-inflammatory markers in activated astrocytes [10]. In addition, we further revealed a novel function of sEH in the regulation of STAT3 activation in activated astrocytes. Based on our findings, the genetic deletion or pharmacological blockade of sEH led to an increase in LPS-induced STAT3 phosphorylation in activated astrocytes, which can be significantly attenuated by the inhibition of STAT3 using stattic in LPS-activated astrocytes. These data suggest that the STAT3-mediated astrocyte activation plays an important role in the regulation of LPS-activated astrocyte activation, whereas the pro-inflammatory properties of STAT3 are likely to be at least partly negatively regulated by $\mathrm{sEH}$. In addition to its pro-inflammatory properties, many studies have reported that STAT3 activity can be anti-inflammatory and might promote the expressions of many antiinflammatory genes, resulting in the indirect inhibition of pro-inflammatory gene expression in dendritic cells or macrophages $[39,40]$. Indeed, our data show that stattic alone triggered the expression of TNF $\alpha$ and IL-6 in astrocytes at resting condition. It is conceivable to speculate that the upregulation of the pro-inflammatory cytokines by stattic in astrocytes at basal levels might result from the blockade of the anti-inflammatory mode of STAT3. However, the possibility that effects of stattic on the expression of pro-inflammatory cytokines are independent of STAT3 activation cannot be excluded. Taken together, the sEH-mediated STAT3 inhibition may play multiple roles in the regulation of astrocyte activation. Further research is needed to clarify how sEH interacts with STAT3 and how the SEH-mediated STAT3 inhibition contributes to the pathogenesis of AD.

\section{Conclusions}

In conclusion, we demonstrated, for the first time, that the deletion of $\mathrm{sEH}$ in astrocytes was pro-inflammatory, whereas the overexpression of $\mathrm{sEH}$ suppressed the immune response. The underlying mechanism may be at least partly attributed to the regulation of STAT3 activation. The sEH-mediated STAT3 inhibition in a cellular context suggests that astrocytic sEH may play an important role in the regulatory machinery of pro-inflammatory response in activated astrocytes during the progression of the brain diseases. Revealing the underlying mechanisms through which sEH orchestrates STAT3 activity and developing means to manipulate astrocytic sEH expression could facilitate the future development of treatment for brain diseases.

\section{Additional file}

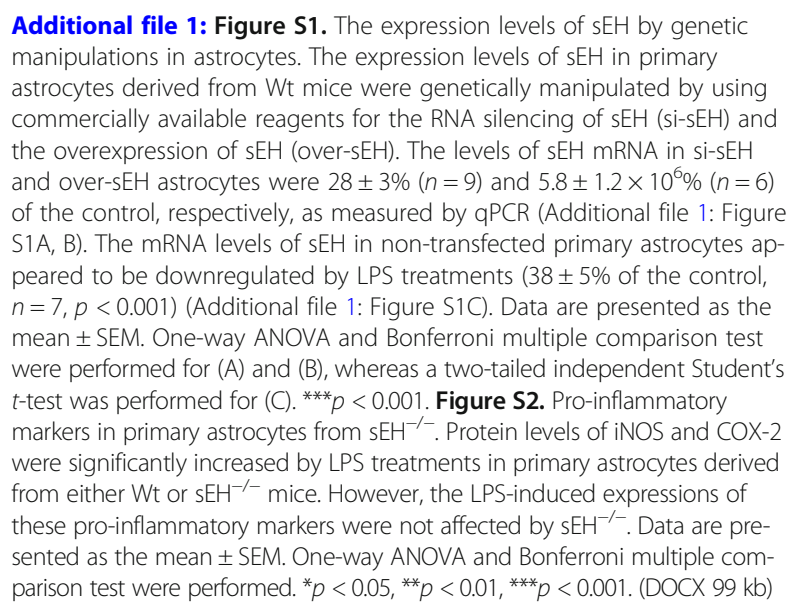

Additional file 1: Figure S1. The expression levels of sEH by genetic manipulations in astrocytes. The expression levels of sEH in primary astrocytes derived from Wt mice were genetically manipulated by using commercially available reagents for the RNA silencing of sEH (si-sEH) and the overexpression of sEH (over-sEH). The levels of sEH mRNA in si-sEH and over-sEH astrocytes were $28 \pm 3 \%(n=9)$ and $5.8 \pm 1.2 \times 10^{6} \%(n=6)$ of the control, respectively, as measured by GPCR (Additional file 1: Figure $\mathrm{S} 1 \mathrm{~A}, \mathrm{~B})$. The mRNA levels of sEH in non-transfected primary astrocytes appeared to be downregulated by LPS treatments ( $38 \pm 5 \%$ of the control, $n=7, p<0.001$ ) (Additional file 1: Figure S1C). Data are presented as the mean \pm SEM. One-way ANOVA and Bonferroni multiple comparison test were performed for (A) and (B), whereas a two-tailed independent Student's $t$-test was performed for (C). ${ }^{* *} p<0.001$. Figure S2. Pro-inflammatory markers in primary astrocytes from $\mathrm{sEH}^{-1-}$. Protein levels of iNOS and COX-2 were significantly increased by LPS treatments in primary astrocytes derived from either Wt or $\mathrm{SEH}^{-1-}$ mice. However, the LPS-induced expressions of these pro-inflammatory markers were not affected by $\mathrm{sEH}^{-/-}$. Data are presented as the mean \pm SEM. One-way ANOVA and Bonferroni multiple comparison test were performed. ${ }^{*} p<0.05,{ }^{* *} p<0.01,{ }^{* * *} p<0.001$. (DOCX $99 \mathrm{~kb}$ )

\section{Abbreviations}

AD: Alzheimer's disease; AFC: N-acetyl-S-farnesyl-L-cysteine; APP/ PS1: APPswe/PSEN1dE9, an Alzheimer's disease mouse model; AUDA: 12-(3adamantan-1-yl-ureido)-dodecanoic acid; $A \beta$ : $\beta$-amyloid; COX2: Cyclooxygenase 2; ECL: Electrochemiluminescence;

EETs: Epoxyeicosatrienoic acids; GFAP: Glial fibrillary acidic protein; HETEs: Hydroxyeicosatetraenoic acids; IL-6: Interleukin-6; iNOS: Inducible nitric oxide; JAK2: Janus kinase 2; LPS: Lipopolysaccharides; NFkB: Nuclear factor 
kappa B; NO: Nitric oxide; over-sEH: Overexpression of sEH; sEH: Soluble epoxide hydrolase; $\mathrm{SEH}^{-/-}$: SEH knockout; si-sEH: Silencing of sEH; STAT3: Signal transducer and activator of transcription 3; TNFa: Tumor necrosis factor a; Wt: Wild-type

\section{Acknowledgements}

This work was supported by the grants NP-PP03 from the National Health Research Institutes, Central Government S\&T grant, Taiwan (107-1901-01-1002), and by the grant MOST 105-2320-B400-001. This work was also financially supported by the Brain Research Center, National Yang-Ming University from The Featured Areas Research Center Program within the framework of the Higher Education Sprout Project by the Ministry of Education (MOE) in Taiwan (107BRC-B405, 108BRC-B404). The authors thank Prof. Tzong-Shyuan Lee at National Yang-Ming University for providing sEH-/- mice and acknowledge the technical assistance by J.C. Lu and W.T. Zhang and the technical support provided by the Imaging Core Facility of Nanotechnology of the UST-YMU. All authors declare no conflicts of interest.

\section{Authors' contributions}

$\mathrm{C}-\mathrm{CH}, \mathrm{Y}-\mathrm{HL}, \mathrm{H}-\mathrm{JT}$, and F-SS designed research. C-CH, P-CH, Y-TH, C-CL, JJL, and F-SS performed research. C-CH, Y-MK, P-CH, JJL, and F-SS analyzed data. $\mathrm{Y}-\mathrm{HL}$ and F-SS wrote the paper. All authors read and approved the final manuscript.

\section{Funding}

NP-PP03 from National Health Research Institutes, Central Government S\&T grant, Taiwan (107-1901-01-10-02), and MOST 105-2320-B400-001.

\section{Availability of data and materials}

Not applicable

\section{Ethics approval and consent to participate}

Not applicable

Consent for publication

Not applicable

\section{Competing interests}

The authors declare that they have no competing interests.

\section{Publisher's Note}

Springer Nature remains neutral with regard to jurisdictional claims in published maps and institutional affiliations.

\section{Author details}

'Institute of Physiology, Brain Research Center, National Yang-Ming University, Taipei, Taiwan, Republic of China. ${ }^{2}$ Institute of Neuroscience, Brain Research Center, National Yang-Ming University, Taipei, Taiwan, Republic of China. ${ }^{3}$ Center for Neuropsychiatric Research, National Health Research Institutes, No.35 Keyan Road, Zhunan Town, Miaoli County 350, Taiwan, Republic of China.

\section{Received: 28 January 2019 Accepted: 21 May 2019}

\section{Published online: 08 June 2019}

\section{References}

1. Li C, Zhao R, Gao K, Wei Z, Yin MY, Lau LT, Chui D, Yu AC. Astrocytes: implications for neuroinflammatory pathogenesis of Alzheimer's disease. Curr Alzheimer Res. 2011;8:67-80.

2. Zhao J, O'Connor T, Vassar R. The contribution of activated astrocytes to Abeta production: implications for Alzheimer's disease pathogenesis. $J$ Neuroinflammation. 2011:8:150

3. Aarum J, Sandberg K, Haeberlein SL, Persson MA. Migration and differentiation of neural precursor cells can be directed by microglia. Proc Natl Acad Sci U S A. 2003;100:15983-8.

4. Bilbo SD, Schwarz JM. Early-life programming of later-life brain and behavior: a critical role for the immune system. Front Behav Neurosci. 2009:3:14.

5. Ben Achour S, Pascual O. Glia: the many ways to modulate synaptic plasticity. Neurochem Int. 2010;57:440-5.

6. Agostinho P. Cunha RA, Oliveira C. Neuroinflammation, oxidative stress and the pathogenesis of Alzheimer's disease. Curr Pharm Des. 2010;16:2766-78.
7. Verri M, Pastoris O, Dossena M, Aquilani R, Guerriero F, Cuzzoni G, Venturini L, Ricevuti G, Bongiorno Al. Mitochondrial alterations, oxidative stress and neuroinflammation in Alzheimer's disease. Int J Immunopathol Pharmacol. 2012:25:345-53.

8. Heneka MT, Carson MJ, El Khoury J, Landreth GE, Brosseron F, Feinstein DL, Jacobs AH, Wyss-Coray T, Vitorica J, Ransohoff RM, et al. Neuroinflammation in Alzheimer's disease. Lancet Neurol. 2015;14:388-405.

9. Bronzuoli MR, lacomino A, Steardo L, Scuderi C. Targeting neuroinflammation in Alzheimer's disease. J Inflamm Res. 2016;9:199-208.

10. O'Callaghan JP, Kelly KA, VanGilder RL, Sofroniew MV, Miller DB. Early activation of STAT3 regulates reactive astrogliosis induced by diverse forms of neurotoxicity. PLoS One. 2014;9:e102003.

11. Peng H, Sun L, Jia B, Lan X, Zhu B, Wu Y, Zheng J. HIV-1-infected and immuneactivated macrophages induce astrocytic differentiation of human cortical neural progenitor cells via the STAT3 pathway. PLoS One. 2011;6:e19439.

12. Beurel E, Jope RS. Lipopolysaccharide-induced interleukin-6 production is controlled by glycogen synthase kinase-3 and STAT3 in the brain. J Neuroinflammation. 2009;6:9.

13. Fuller S, Munch G, Steele M. Activated astrocytes: a therapeutic target in Alzheimer's disease? Expert Rev Neurother. 2009;9:1585-94.

14. Gonzalez-Reyes RE, Nava-Mesa MO, Vargas-Sanchez K, Ariza-Salamanca D, Mora-Munoz L. Involvement of astrocytes in Alzheimer's disease from a neuroinflammatory and oxidative stress perspective. Front Mol Neurosci. 2017;10:427.

15. Zamanian JL, Xu L, Foo LC, Nouri N, Zhou L, Giffard RG, Barres BA. Genomic analysis of reactive astrogliosis. J Neurosci. 2012;32:6391-410.

16. Voskuhl RR, Peterson RS, Song B, Ao Y, Morales LB, Tiwari-Woodruff S, Sofroniew MV. Reactive astrocytes form scar-like perivascular barriers to leukocytes during adaptive immune inflammation of the CNS. J Neurosci. 2009:29:11511-22.

17. Myer DJ, Gurkoff GG, Lee SM, Hovda DA, Sofroniew MV. Essential protective roles of reactive astrocytes in traumatic brain injury. Brain. 2006;129:2761-72.

18. Wyss-Coray T, Loike JD, Brionne TC, Lu E, Anankov R, Yan F, Silverstein SC, Husemann J. Adult mouse astrocytes degrade amyloid-beta in vitro and in situ. Nat Med. 2003;9:453-7.

19. Vehmas AK, Kawas CH, Stewart WF, Troncoso JC. Immune reactive cells in senile plaques and cognitive decline in Alzheimer's disease. Neurobiol Aging. 2003;24:321-31.

20. Koistinaho M, Lin S, Wu X, Esterman M, Koger D, Hanson J, Higgs R, Liu F, Malkani S, Bales KR, Paul SM. Apolipoprotein E promotes astrocyte colocalization and degradation of deposited amyloid-beta peptides. Nat Med. 2004;10:719-26.

21. Chun H, Lee CJ. Reactive astrocytes in Alzheimer's disease: a double-edged sword. Neurosci Res. 2018;126:44-52.

22. Shie FS, Woltjer RL. Manipulation of microglial activation as a therapeutic strategy in Alzheimer's disease. Curr Med Chem. 2007;14:2865-71.

23. Simpkins AN, Rudic RD, Schreihofer DA, Roy S, Manhiani M, Tsai HJ, Hammock BD, Imig JD. Soluble epoxide inhibition is protective against cerebral ischemia via vascular and neural protection. Am J Pathol. 2009;174:2086-95

24. Shaik JS, Ahmad M, Li W, Rose ME, Foley LM, Hitchens TK, Graham SH, Hwang SH, Hammock BD, Poloyac SM. Soluble epoxide hydrolase inhibitor trans-4-[4-(3-adamantan-1-yl-ureido)-cyclohexyloxy]-benzoic acid is neuroprotective in rat model of ischemic stroke. Am J Physiol Heart Circ Physiol. 2013;305:H1605-13.

25. Zhang W, Otsuka T, Sugo N, Ardeshiri A, Alhadid YK, lliff JJ, DeBarber AE, Koop DR, Alkayed NJ. Soluble epoxide hydrolase gene deletion is protective against experimental cerebral ischemia. Stroke. 2008;39:2073-8.

26. Wu C-H, Shyue S-K, Hung T-H, Wen S, Lin C-C, Chang C-F, Chen S-F. Genetic deletion or pharmacological inhibition of soluble epoxide hydrolase reduces brain damage and attenuates neuroinflammation after intracerebral hemorrhage. J Neuroinflammation. 2017;14:230.

27. Tu R, Armstrong J, Lee KS, Hammock BD, Sapirstein A, Koehler RC. Soluble epoxide hydrolase inhibition decreases reperfusion injury after focal cerebral ischemia. Sci Rep. 2018;8:5279.

28. Zarriello S, Tuazon JP, Corey S, Schimmel S, Rajani M, Gorsky A, Incontri D, Hammock BD, Borlongan CV. Humble beginnings with big goals: small molecule soluble epoxide hydrolase inhibitors for treating CNS disorders. Prog Neurobiol. 2019;172:23-39.

29. Panigrahy D, Greene ER, Pozzi A, Wang DW, Zeldin DC. EET signaling in cancer. Cancer Metastasis Rev. 2011;30:525-40. 
30. Cronin A, Mowbray S, Durk H, Homburg S, Fleming I, Fisslthaler B, Oesch F, Arand $\mathrm{M}$. The N-terminal domain of mammalian soluble epoxide hydrolase is a phosphatase. Proc Natl Acad Sci U S A. 2003;100:1552-7.

31. Enayetallah AE, French RA, Thibodeau MS, Grant DF. Distribution of soluble epoxide hydrolase and of cytochrome P450 2C8, 2C9, and $2 \mathrm{~J} 2$ in human tissues. J Histochem Cytochem. 2004:52:447-54.

32. Sura P, Sura R, Enayetallah AE, Grant DF. Distribution and expression of soluble epoxide hydrolase in human brain. J Histochem Cytochem. 2008;56: 551-9.

33. Hung YW, Hung SW, Wu YC, Wong LK, Lai MT, Shih YH, Lee TS, Lin YY. Soluble epoxide hydrolase activity regulates inflammatory responses and seizure generation in two mouse models of temporal lobe epilepsy. Brain Behav Immun. 2015:43:118-29.

34. Maragakis NJ, Rothstein JD. Mechanisms of disease: astrocytes in neurodegenerative disease. Nat Clin Pract Neurol. 2006;2:679-89.

35. Yamanaka K, Chun SJ, Boillee S, Fujimori-Tonou N, Yamashita H, Gutmann DH, Takahashi R, Misawa H, Cleveland DW. Astrocytes as determinants of disease progression in inherited amyotrophic lateral sclerosis. Nat Neurosci. 2008:11:251-3.

36. Anderson MA, Ao Y, Sofroniew MV. Heterogeneity of reactive astrocytes. Neurosci Lett. 2014;565:23-9.

37. Mathur R, Ince PG, Minett T, Garwood CJ, Shaw PJ, Matthews FE, Brayne C, Simpson JE, Wharton SB, Function MRCC, Ageing Neuropathology Study G. A reduced astrocyte response to beta-amyloid plaques in the ageing brain associates with cognitive impairment. PLoS One. 2015;10:e0118463.

38. Wyss-Coray T, Mucke L. Inflammation in neurodegenerative disease--a double-edged sword. Neuron. 2002;35:419-32.

39. Murray PJ. The primary mechanism of the IL-10-regulated antiinflammatory response is to selectively inhibit transcription. Proc Natl Acad Sci U S A. 2005;102:8686-91.

40. Hutchins AP, Diez D, Miranda-Saavedra D. The IL-10/STAT3-mediated antiinflammatory response: recent developments and future challenges. Brief Funct Genomics. 2013:12:489-98.

Ready to submit your research? Choose BMC and benefit from:

- fast, convenient online submission

- thorough peer review by experienced researchers in your field

- rapid publication on acceptance

- support for research data, including large and complex data types

- gold Open Access which fosters wider collaboration and increased citations

- maximum visibility for your research: over $100 \mathrm{M}$ website views per year

At $\mathrm{BMC}$, research is always in progress.

Learn more biomedcentral.com/submissions 